\title{
Quantification of Protein-Ligand Interaction Using Supported Lipid Bilayer Assisted Biosensors
}

\author{
Donggeun Lee ${ }^{1,2}$, Youngmo Jung ${ }^{1,3}$, Taikjin Lee ${ }^{1}$, Jae Hun Kim¹, Seok Lee ${ }^{1}$, Sang-Kook Han², Yong-Sang \\ Ryu', Chulki Kim ${ }^{1}$ \\ ${ }^{1}$ Sensor System Research Center, Korea Institute of Science and Technology \\ 5, Hwarang-ro 14-gil, Seongbuk-gu, Seoul, 02792, Republic of Korea \\ chulki.kim@kist.re.kr \\ ${ }^{2}$ Department of Electrical \&Electronic Engineering, Yonsei University \\ 50 Yonsei-ro Seodaemun-gu, Seoul, 03722, Republic of Korea \\ ${ }^{3}$ Department of Mechanical Engineering, Yonsei University \\ 50 Yonsei-ro Seodaemun-gu, Seoul, 03722, Republic of Korea
}

\section{Extended Abstract}

Quantification of kinetics of protein interactions has been a fundamental challenge in biophysics and biotechnology ${ }^{[1],[2]}$. To investigate the binding kinetics on a cell membrane rigorously, active ligands should be prepared in a controlled environment in terms of the number of binding sites and its kind. Conventional binding assays using ligand immobilization techniques with glue-like layers still have problems typically related to ligand denaturalization and non-specific binding. To demonstrate monitoring real-time binding kinetics between proteins and ligands, we introduce a supported lipid bilayer (SLB) to model the binding kinetics. The role of the supported lipid bilayer here is three-fold: accurate control over the binding sites, structural formation of receptors, and reducing non-specific bindings effectively. We adopted a field effective transistor device capable of reliable observation of protein interactions via its modulated current responses. The binding sites and rate constants of the protein-ligand pair interaction are determined by monitoring the real-time reaction kinetics, demonstrating the possible quantification of protein interactions with a detection limit of picomolar concentration and association constant was about $1 \times 10^{9} \mathrm{M}^{-1}$ using SLB assisted biosensors.

\section{References}

[1] X. Duan, "Quantification of the affinities and kinetics of protein interactions using silicon nanowire biosensors," Nat. Nano., vol. 7, no. 82, pp.401-407, 2012.

[2] S. Xu. "Real-time reliable determination of binding kinetics of DNA hybridization using a multi-channel graphene biosensor," Nat. Comm., vol. 8, no. 14902, pp.1-10, 2017. 